\title{
Solar forced variations of terrestrial high energy particle environment as seen by RESIK PIN detectors on CORONAS-F
}

\author{
M. Kowalinski ${ }^{1}$, Z. Kordylewski ${ }^{1}$, J. Sylwester ${ }^{1}$, W. Trzebinski $^{1}$ \\ and D. Lisin ${ }^{2}$
}

${ }^{1}$ Space Research Centre, Polish Academy of Sciences, Wroclaw ul. Kopernika 11, Poland email: mk@cbk.pan.wroc.pl

${ }^{2}$ IZMIRAN, Russian Academy of Sciences

\begin{abstract}
RESIK is the bent crystal spectrometer aboard the CORONAS-F satellite. It is equipped with four PIN diode detectors. These detectors were used in order to detect background counts due to energetic particle contamination present within polar regions and SAA belts. At altitudes above $300 \mathrm{~km}$ (Coronas-F altitude is about $500 \mathrm{~km}$ ) the population of charged particles trapped by the Earth's magnetic field consists of protons (energies between $100 \mathrm{keV}$ and several hundred $\mathrm{MeV}$ ) and electrons (energies between few tens of $\mathrm{keV}$ and $10 \mathrm{MeV}$ ). The construction of RESIK PIN diode detectors allows to sense particles with the energy above $1 \mathrm{MeV}$. We present diagrams illustrating the coupling of the Coronas-F particle environment and selected proxies of solar activity. The data shown have been collected during initial phase of mission.
\end{abstract}

\section{Introduction}

Variations in certain manifestations of solar activity such as solar wind and/or transient phenomena like interplanetary magnetic fields boundary crossings, erupting filaments, coronal mass ejection, presence of equatorial coronal holes, may cause disturbances in the state of Earth magnethosphere. The effects of these disturbances are especially noticeable in polar regions and possibly in the South Atlantic Anomaly (SAA). The SAA is the region of a lower geomagnetic field extending above Brazil and south Atlantic. In this zone, which extends down to low Earth orbit (LEO) altitudes, the energetic particle flux is significantly increased. In the SAA region the LEO satellites including ISS and shuttles, are exposed to increased levels of harmfull radiation. Polar cap regions with increased energetic particle population are also dangerous to the unmanned satellite systems. In this paper we analyse the varaiation in the particle environment in the auroral cap regions and SAA from the records of RESIK PIN diode sensors placed aboard in order to detect regions and times where/when the high voltage in RESIK proportional detectors should be switched off. Signals from these PIN sensors has been analysed aboard RESIK in order to switch-off the high voltage from the primary large area proportional detectors (measuring solar soft X-ray spectra) during passages through regions of increased energetic particle density.

\section{Observation and results}

The PIN sensors have been collecting data from September 2001 to September 2003. From various available, we have found the best correlation $(\mathrm{R}=0.97$, left part in the Figure) between PIN sensor rates and MKL proton rates for high-energy protons $(\mathrm{Ep}>26 \mathrm{MeV})$. Time variations of RESIK PIN fluency, as considered in three separate 

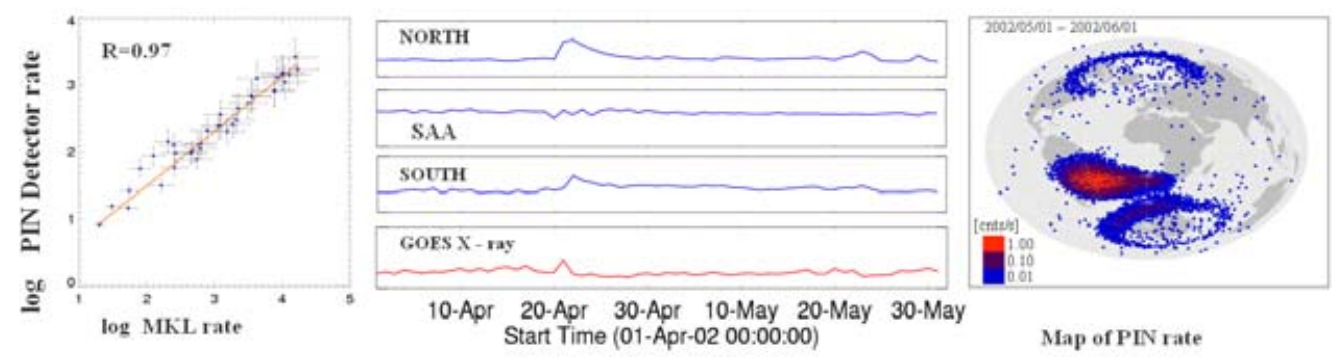

Figure 1. Left: The correlation diagram showing direct correspondence between the rates seen by RESIK PIN sensors and the rate measured by the MKL instrument. Centre: Plots of time variation of the PIN sensor rates in the three regions of geomagnetic environment (North and South polar caps and SAA) atop variations of GOES-10 X-radiation fluxes for the corresponding times in April-May 2002. Right: Map showing the location of regions with increased rates of the PIN sensors as determined from data collected over May 2002.

regions of the magnetosphere (South polar oval, SAA and North polar oval regions) are shown in the middle. The map in the Figure shows areas of higher PIN rates as seen in May 2002.

In both polar regions, increased PIN rates are distinctly observed to follow certain flares seen on the GOES soft X-ray plot. Th SAA region shows up also some activity-associated variability also, however with a much smaller amplitude. Flare related increases in the SAA rates are observed to be much more delayed in time with respect to polar regions increases. Overall observed fluctuations in the particle rate recorded within SAA are approximately by an order of magnitude, with the peak levels few times the average rate. This observed significant SAA changes affect all LEO satellites including ISS.

\section{Acknowledgements}

RESIK (PI - J. Sylwester), is a common project between NRL (USA), MSSL and RAL (UK), IZMIRAN (Russia) and SRC (Poland). M.K., Z.K., J.S. and W.T. acknowledge support from grant 2.P03D.002.22 of the Polish Committee for Scientific Research.

\section{References}

Martin V. Zombeck 1982 Handbook of Space Astronomy and Astrophysics , 154-159. 